\title{
Clonal evolution in chronic lymphocytic leukemia is scant in relapsed but accelerated in refractory cases after chemo(immune) therapy
}

Haematologica 2022

Volume 107(3):604-614

\section{Correspondence:}

PETER LICHTER

Peter.Lichter@dkfz-heidelberg.de

STEPHAN STILGENBAUER

Stephan.Stilgenbauer@uniklinik-ulm.de

Received: July 7, 2020.

Accepted: February 26, 2021.

Pre-published: March 11, 2021.

https://doi.org/10.3324/haematol.2020.265777

(C)2022 Ferrata Storti Foundation

Material published in Haematologica is covered by copyright. All rights are reserved to the Ferrata Storti Foundation. Use of published material is allowed under the following terms and conditions:

https://creativecommons.org/licenses/by-nc/4.0/legalcode. Copies of published material are allowed for personal or internal use. Sharing published material for non-commercial purposes is subject to the following conditions:

https://creativecommons.org/licenses/by-nc/4.0/legalcode, sect. 3. Reproducing and sharing published material for commercial purposes is not allowed without permission in writing from the publisher.

\section{Marc Zapatka, ${ }^{1 *}$ Eugen Tausch, ${ }^{2 *}$ Selcen Öztürk, ${ }^{1}$ Deyan Yordanov Yosifov, ${ }^{2,3}$ Martina Seiffert, ${ }^{1}$ Thorsten Zenz, ${ }^{4}$ Christof Schneider, ${ }^{2}$ Johannes Bloehdorn, ${ }^{2}$ Hartmut Döhner, ${ }^{2}$ Daniel Mertens, ${ }^{2,3}$ Peter Lichter ${ }^{1}$ and Stephan Stilgenbauer ${ }^{2}$}

${ }^{1}$ Division of Molecular Genetics, German Cancer Research Center, Heidelberg, Germany; ${ }^{2}$ Department of Internal Medicine III, Ulm University Hospital, UIm, Germany;

${ }^{3}$ Mechanisms of Leukemogenesis, German Cancer Research Center (DKFZ), Heidelberg, Germany and ${ }^{4}$ University Hospital, University of Zürich, Zürich, Switzerland

${ }^{*} M Z$ and $E T$ contributed equally as co-first authors.

\section{ABSTRACT}

C lonal evolution is involved in the progression of chronic lymphocytic leukemia (CLL). In order to link evolutionary patterns to different disease courses, we performed a long-term longitudinal mutation profiling study of CLL patients. Tracking somatic mutations and their changes in allele frequency over time and assessing the underlying cancer cell fraction revealed highly distinct evolutionary patterns. Surprisingly, in long-term stable disease and in relapse after long-lasting clinical response to treatment, clonal shifts are minor. In contrast, in refractory disease major clonal shifts occur although there is little impact on leukemia cell counts. As this striking pattern in refractory cases is not linked to a strong contribution of known CLL driver genes, the evolution is mostly driven by treatment-induced selection of sub-clones, underlining the need for novel, non-genotoxic treatment regimens.

\section{Introduction}

Cancer can be conceptualized as an evolutionary process within a given organism. ${ }^{1,2}$ By increasing the fitness of cancer cells, mutations enable sub-clones to outcompete non-malignant cells and less adapted cancer cell clones. Furthermore, clonal evolution allows the selection of cell populations that are resistant to therapy or responsible for disease recurrence. For some tumor entities like acute myeloid leukemia (AML), the concept of cancer initiating cells seems to account for tumor relapses without further genetic evolution. For other malignancies however, it is more likely that additional mutations play a crucial role in tumor recurrence. This is also true for chronic lymphocytic leukemia (CLL), where progression and clonal evolution have been analyzed in the context of treatment induced genetic changes. ${ }^{3,4}$

Clinically, CLL is characterized by a highly variable course. The survival time of patients varies between months and decades. Often patients remain untreated for many years until clinical symptoms require therapeutic intervention. ${ }^{5}$ Despite high rates of initial treatment response, a major clinical challenge is the occurrence of refractory disease that does not respond to treatment. Refractory cases are often characterized by a deletion and/or a mutation in the tumor suppressor gene TP53 located on the short arm of chromosome 17 (del17p/TP53mut). Although a number of recurrently mutated genes were identified in CLL that are of prognostic relevance $^{4,-9}$ del17p/TP53mut remains the strongest adverse prognostic factor for progression-free and overall survival in CLL.,10,11 The incidence of mutated or deleted TP53 is below 3\% in Binet A stage CLL representing cases with good prognosis or in the pre-malignant monoclonal B-cell lymphocytosis (MBL) state, but increases to $12 \%$ at time of first treatment initiation, and to more than $37 \%$ in chemotherapy refractory cohorts. ${ }^{8,12,13}$ Despite this increase in cases with mutated or deleted TP53 at later disease stages, clonal evolution has been considered rare in CLL. 
Early cytogenetic and molecular cytogenetic studies reported unequivocal evidence for occurrence of clonal evolution in CLL, albeit rare..$^{14-17}$ More recently, high-resolution microarray and next-generation sequencing (NGS) based approaches were applied to track subclonal heterogeneity and clonal evolution in CLL. Based on a single nucleotide polymorphism (SNP) micro-array analysis of pretreatment and relapsed samples from 42 patients, DNA copy number variations (CNV) were reported that expand or newly occur at relapse. ${ }^{18}$ The respective genomic regions contain candidate driver genes of relapse and/or chemotherapy resistance. Somatic mutation profiling of CLL by NGS revealed recurrent gene alterations ${ }^{19}$ and confirmed molecular heterogeneity. ${ }^{20}$ The comprehensive analysis of 149 CLL cases allowed to distinguish clonal (MYD88, trisomy 12, and $\operatorname{del}(13 q)$ ) and subclonal (SF3B1 and TP53) driver mutations ${ }^{20}$ and this order was validated by the same group in a huge clinical study. ${ }^{4}$ While a considerable number of driver genes and recurrent genomic alterations were identified via whole-exome sequencing (WES) analysis of a cumulative number of more than 1,000 CLL patients, there are only few studies that decipher changes of drivers over the course of disease. Mutation profiling of three CLL patients over time indicated heterogeneous clonal evolution patterns. ${ }^{21}$ By a similar approach, ten of 12 CLL cases treated with chemotherapy were shown to undergo evolution of sub-clones with respective driver mutations (SF3B1 and TP53), while this was detected in only one of six cases that were not treated. ${ }^{22}$ While one study reported that clonal composition remained stable at disease progression and relapse ${ }^{23}$ another study referred that 13 of 28 sequentially sampled cases underwent genetic change of $>20 \%$ with nine of them (but none of the nonevolving cases) also displaying epigenetic evolution. ${ }^{24}$

A number of deep sequencing studies focused on a targeted panel for candidate genes in CLL and provided evidence of clonal outgrowth over time i.e., of TP53 after treatment. ${ }^{25-27}$ Despite that, their major focus was on untreated patient samples and the response to therapy was not considered as a predictor of evolution. In addition, targeted analysis of a restricted number of drivers can give an idea of clonal rigidity, but fail to show emergence and outgrowth of new subclones characterized by variants not covered with the panel. A similar approach considering aberrations in addition to known driver mutations deciphered the history of these alterations by integrating longitudinal and cross-sectional data in 70 patients. ${ }^{28}$ While the distinction of evolutionary early and late events showed a similar pattern to Landau et al., again the association with patient outcome was not addressed. The biggest WES cohort with sequential sampling in CLL included 59 patients from CLL8 with samples before and after relapse to FC/FCR (fludarabine, cyclophosphamide, rituximab) showing changes of cell fractions characterized by specific drivers as well as linear versus branched evolution patterns in 57 of 59 cases. ${ }^{4}$ However, this group consisted only of relapsed cases with a missing control of refractory and long-term untreated patients. Due to the fact, that again type and duration of response were not considered as parameters, a link between treatment, outcome and dynamic genomic changes in CLL is barely explored. Although a connection of response to therapy and dynamic genomic changes is plausible, it remains unclear how clonal evolution is linked to long-term stable, to relapsed or to refractory disease.
In order to elucidate the clonal evolution of CLL cell populations in the presence or absence of therapy, we performed a long-term longitudinal mutation profiling study of a multifarious cohort of CLL patients with a well annotated patient history.

Aberrant TP53 dictates the clinical course of the disease, it is a key driver of acquired resistance and potentially supersedes other parameters. Therefore, we excluded patients with del17p or mutated TP53 status at baseline as we presumed that these patients had acquired the most relevant evolution marker already. Samples were obtained at different time points before and after treatment in three different clinical groups: i) long-term untreated cases with stable disease and no need for treatment over at least 4 years, ii) relapsed cases with durable response to therapy of at least 2 years, and iii) refractory cases without response to treatment (stable disease [SD], progressive disease $[\mathrm{PD}]$ ) or cases that progressed with requirement of a subsequent therapy within 1 year. WES was performed and data were subsequently partially validated by targeted resequencing of identified mutations.

\section{Methods}

\section{Sample collection}

We compiled an inventory of CLL patient samples before and after treatment and sequenced tumor and non-tumor control DNA (25 patients and 54 tumor samples including 21 patients with baseline samples prior to any therapy). Our inclusion criteria were: (i) no del17p or mutated TP53 status at baseline, (ii) patients fitting to any of the three groups (a) long-term untreated cases with stable disease and no need for treatment over at least 4 years, (b) relapsed cases with durable response to therapy of at least 2 years, and (c) refractory cases without response to treatment $(\mathrm{SD}, \mathrm{PD})$ or cases that progressed with requirement of a subsequent therapy within 1 year.

All patients gave informed consent according to the Helsinki Declaration. Sample acquisition for sequencing purposes was approved by a local Ethics Review Committee (Ethikkommision Ulm University, ethik-kommission@uni-ulm.de, 17.06.2008, 96/08-UBB/se).

Peripheral blood mononuclear cell (PBMC) samples were enriched for tumor (CD19+) and normal CD19-cells using MACS microbead cell separation (Miltenyi Biotec, Bergisch Gladbach, Germany). Genomic DNA was isolated from unsorted and sorted CLL cells using All Prep Kit (Qiagen, Hilden, Germany). Quality and quantity of the purified DNA were assessed with the Qubit dsDNA BR Assay Kit (Lifetech technologies, Carlsbad, CA).

\section{Sequencing}

WES was performed on Illumina HiSeq 2000 machines. Exome libraries were created using the TruSeq Exome Library Prep Kit or Agilent SureSelect enrichment Human Exome V4 Kit according to the manufacturer's protocols. Alignment and variant calling were performed as previously described $\mathrm{in}^{29}$.

\section{Allele frequency changes in patient groups}

Per patient single nucleotide variants (SNV) with genotype change were identified and differences in alternative allele frequency (aAF) calculated between consecutive time points. aAF were clustered per patient into six clusters to give each time point equal weight regardless of the number of SNV detected. Each change in aAF was grouped according to the status (untreated, 
relapse, refractory) at the second time point. Differences in the distribution of the allele frequency changes between the three patient groups were identified using a bootstrapped Kolmogorov-Smirnov test with $n=10,000$.

\section{Copy number variations calling and calculation of absolute copy numbers}

Estimation of the copy number state based on the exome sequencing data was achieved using Varscan 2 on the target regions. ${ }^{30}$ Absolute copy numbers were calculated as previously described. $^{31}$

\section{Calculation of cancer cell fraction}

Cancer cell fractions (CCF) integrating sample purity (estimated by fluorescence-activated cell sorting [FACS]), ploidy inferred from fluorescence in situ hybridization (FISH), copy number states calculated from WES and allelic fraction and coverage of somatic variants were calculated for the patients with available germline samples following the approach previously outlined in $^{20}$.

\section{Estimation of clonal composition by TrAP}

Changes in clonal tumor composition were calculated integrating the CCF at the respective time points using TrAP (tree approach to clonality). ${ }^{32}$

\section{Quantification of DNA methylation and estimation of correlation between time points}

DNA methylation from the first and second time point of ten patient phases (three long-term untreated, two relapsed and five refractory) was assessed by Illumina Infinium HumanMethylation450 BeadChips according to the manufacturer's protocol.

Details on the individual approaches are further described in the Online Supplementary Appendix.

\section{Results}

\section{The clinical course of patients grouped into distinct phases}

The clonal evolution in malignant B-cell populations of CLL patients was studied by longitudinal analyses in a total of 25 patients and 54 tumor samples. For 21 patients, the baseline sample was obtained prior to any CLL therapy, whereas four additional patients were pretreated before enrollment in our study. A common case history in CLL can consist of different phases including an untreated phase with a watch and wait strategy in the beginning followed by one or more therapies with either durable or very short responses or even refractoriness to the ongoing treatment. We observed such clinical phases in our patients throughout their individual medical history. For example, some of the long-term untreated patients required therapy at a later stage (e.g., HU-1-06) and some patients with initially long-lasting response became refractory after a subsequent treatment (e.g., HU-1-11). Therefore, we divided the individual patient histories into different clinical phases rather than using a rigid division of patients into categories. Individuals can go through several of these phases with sampling at the beginning and at the end of each phase. Three clinical disease patterns were distinguished and in total we identified 29 phases: six phases were evaluated as long-term untreated, five as relapsed after initially durable response to therapy, and 18 as treatment refractory. Details of the clinical course of patients and patient phases including treatment, treatment response and sampling, as well as cytogenetic grouping and the immunoglobulin heavy-chain variable region gene (IGHV) mutation status are presented in the Online Supplementary Tables $S 1$ to $S 3$ and in the Online Supplementary Figure S1.

\section{Increased mutation rate is associated with refractory disease}

Identification of mutations was performed by comparative WES of CD19+ enriched PBMC and, as non-malignant control, the sorted CD19-negative fraction of PBMC from the same patient. Over the course of this longitudinal study, no $I G H V$ status switch was identified. In IGHVmutated cases, the major $I G H V$ clone did not change, and $I G H V$ mutations and SNP fingerprinting were used to confirm sample identity.

Based on limited material for sorting of non-neoplastic cells, for 19 of 25 patients a non-tumor control was available for mutation detection. Applying established algorithms $\mathrm{s}^{33}$ for the calling of SNV and small insertions and deletion (Indels), we observed an average of 15.1 mutations per sample (range, 2-36) (Online Supplementary Tables $S 4$ to $S 6$ ). A prediction of the response to therapy was not possible based on mutation numbers, as samples taken before long lasting response to therapy and before refractory disease had similar numbers of mutations (11.3 [range, 1-30] and 15.8 [range, 2-34] respectively $P$-value Mann-Whitney test $P=0.36$; Figure $1 \mathrm{~A}$ and $\mathrm{B}$ ). Samples obtained before any therapy as well as post-therapeutic samples from relapsed patients had the lowest number with 13.5 (range, 2-30) and 13.0 (range, 6-25) mutations in contrast to refractory patients with 17.9 (range, 4-36) mutations, respectively (Figure 1B) (Kruskal Wallis test $P=0.30$ ). We identified 1.5 known driver events per sample with the largest variation and highest number of SNV/Indels in refractory CLL samples. All cases except HU-1-08, HU-1-11, and HU-1-21 harbored SNV/Indels in known or candidate CLL driver genes. ${ }^{4,34}$ Indeed, candidates previously associated with adverse outcome like $B I R C 3, E G R 2$ and $S A M H D$ were identified predominantly in refractory cases, but preceded good response to (chemo)therapy and therefore did not determine outcome (e.g., patients HU-1-19 or HU-1-15). In addition, this study revealed genes that had so far not been associated with CLL but were mutated in more than one of the analyzed patients: MC5R, MYH2, RFX7, ROBO2 and SLITRK5.

\section{Clonal evolution of leukemic cells is dominant in patients with refractory disease}

Clonal evolution was modeled on the basis of single nucleotide variants that were assessed in longitudinal sample collections. FISH analysis with a panel of diagnostic probes ${ }^{10}$ in a subset of samples revealed near diploidy of the neoplastic cells. Interestingly, no changes in cytogenetic aberrations in long-term untreated phases could be identified based on FISH data (Online Supplementary Table S1). Most patients retained their karyotype after treatment, but HU-1-19 acquired a deletion in chromosome $17 \mathrm{p}$. Since neoplastic B-cell content was generally higher than $80 \%$, AF were used as basis for modeling evolution over time. To this aim, SNV were identified that displayed variable AF between the time points of molecular analysis. During long-term untreated phases, AF remained stable, which is in accordance with an unchanged clonal compo- 
A

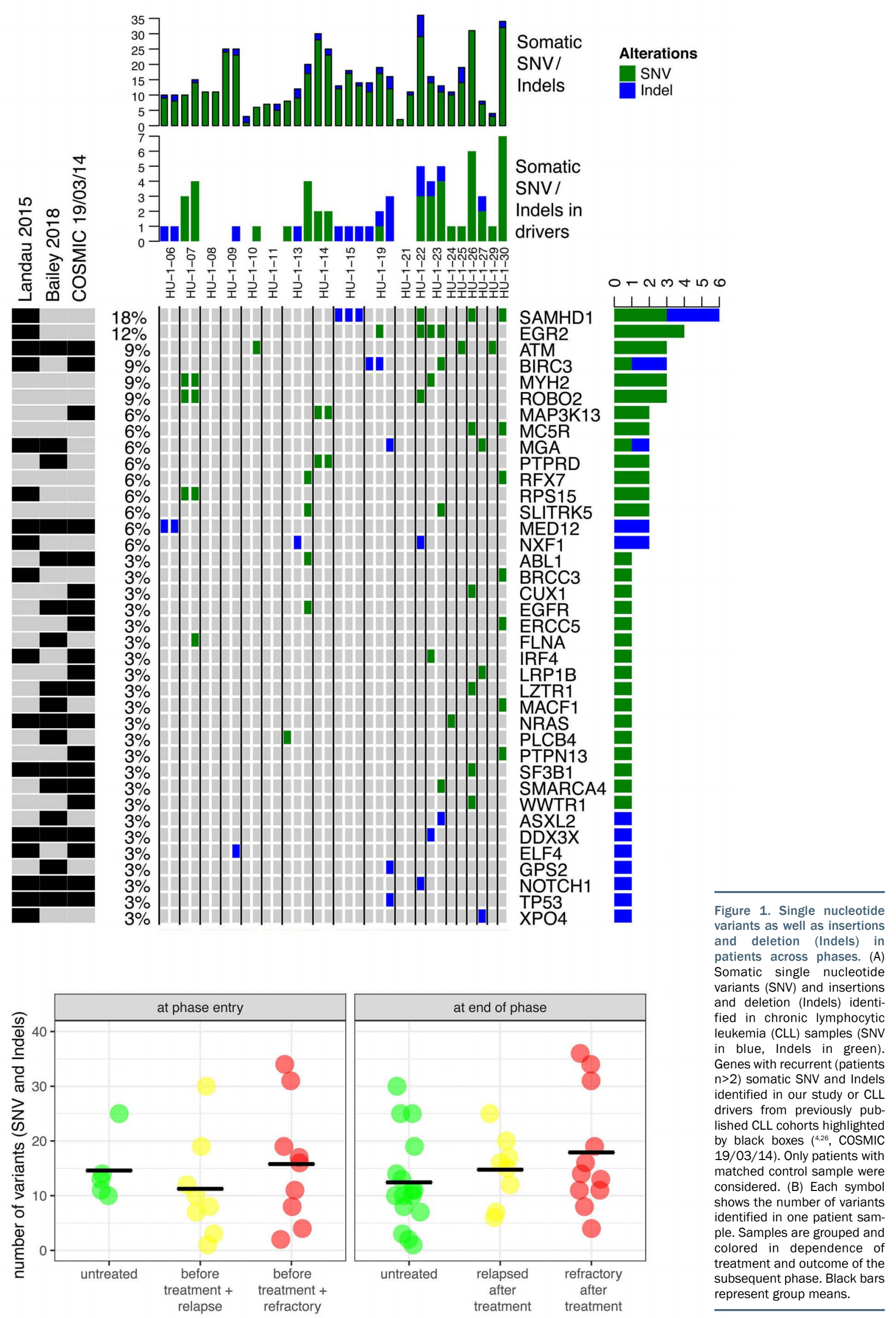


sition. Although one might expect the occurrence of clonal evolution with the acquisition of new variants in tumors with relapse after therapy, we observed the opposite: such clinical phases show the same mutational landscapes both at baseline and at relapse, and major shifts in AF occurred only exceptionally (Figure 2). In sharp contrast, during phases of therapy refractoriness, we found dramatic alterations in clonal composition (Figures 2 and 3). Notably, in refractory phases these increases and reductions in AF occurred within relatively short time intervals (median phase length: refractory 707 days, relapse 2,395 days, untreated 2,088 days, time span refractory phases $v s$. time span untreated, treated and relapsed Mann-Whitney test $P=0.00014$; Online Supplementary Table S1), that were particularly much shorter than the phases in stable or relapsed cases. The high degree of AF changes and the short time window over which these changes occurred indicate marked dynamics in the clonal shift, often notable in tumors that appeared clinically unaffected by therapy (i.e., without remission and subsequent regrowth). These clonal shifts clearly indicate a change in the clonal composition, and strikingly they occur mostly during refractory phases, i.e., during treatment that does not successfully affect the clinical outcome.

We further quantified overall AF changes in the three different groups of clinical phases independent of the further course of disease (Figure 3, changes in AF over time provided in the Online Supplementary Figure S2). Clearly, a substantially higher variation of AF is seen in the samples that reach the therapy refractory phase. Comparison of 28 subsequent time points in 25 patients identified significant differences in the AF changes over time between different types of clinical phases, which indicates that the degree of change in the clonal composition is different in the three clinical groups (Kruskal-Wallis test $P=0.00262$, corrected based on permutation of phase labels). Furthermore, the untreated phases showed a significantly lower AF change over time independent of the extent of time between sampling $(P<0.01$ for untreated vs. relapsed and untreated vs. relapsed/refractory; Online Supplementary Table S7).
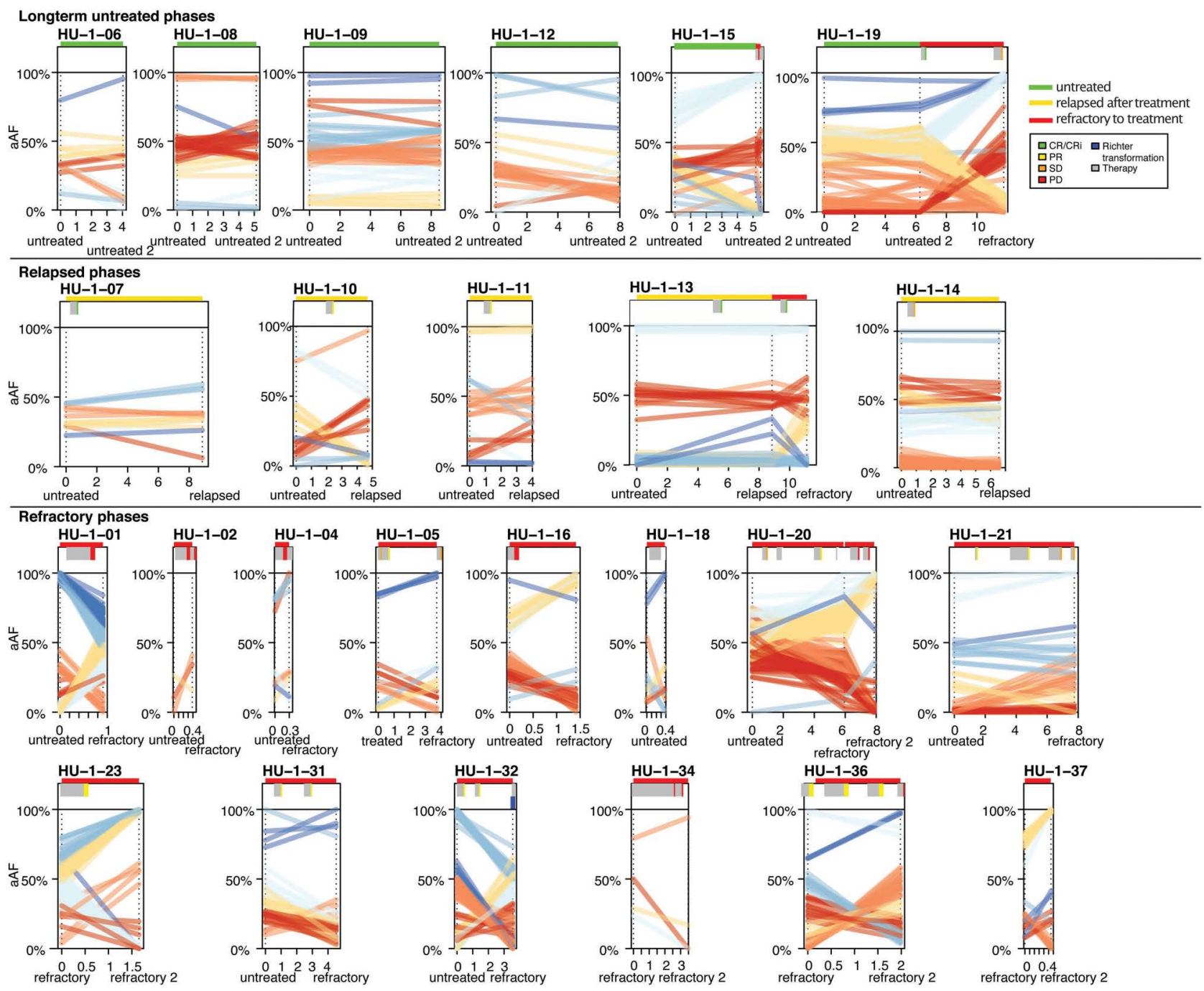

Figure 2. Allele frequency changes during the clinical course of chronic lymphocytic leukemia patients. Overall clinical phase is depicted by colored bars on top (green=untreated, yellow=relapsed after treatment, red=refractory to treatment). The individual treatments and disease progressions are depicted in the first row of each graph with gray bars representing treatment, while green, yellow, orange and red bars indicate the type of treatment response and disease progression. Richter transformation in HU-1-32 (blue) is highlighted in the second row, above the alternative allele frequencies (aAF). aAF changes are colored based on hierarchical clustering of the trajectories following by identifying the six major clusters (R function cutree). $y$-axis: indicates allele frequencies, $x$-axis: indicates time course in years. 
Clonal expansion or reduction of individual sub-clones over time and with treatment

Integrating tumor purity, copy number state (Online Supplementary Figure S3) and AF, we inferred the CCF (Online Supplementary Table S8) affected by individual mutations as described before. ${ }^{4}$ In some patients and phases, known cancer drivers listed in the COSMIC mutation database could be linked to the changes in CCF (Online Supplementary Figure S4; gene symbols from COSMIC highlighted in purple). For example, HU-1-13 showed a mutation in the cancer driver EGFR only in the untreated and refractory sample (CCF 7\% coverage 33 and CCF $66 \%$, coverage 57) (Online Supplementary Figure S4). The mutation was undetected (coverage 48 ) at relapse indicating a reduction of this clone below the detection limit at relapse. Furthermore, the fraction of cells carrying an ANO1 mutation steadily increased from $0 \%$ over $2.3 \%$ to $22.0 \%$ in the refractory sample (coverage 48,42 and 41 ). Interestingly, the major clone present at the relapse and characterized by an NLRP13 mutation (CCF $28.5 \%$, coverage 21) was not detected any more in the refractory sample (coverage 25), indicating that this clone was lost during treatment or during progress.

HU-1-19 displayed similar shifts albeit with a different clonal composition, but also with elimination of a clone after treatment. The EGR2 variant changed from $2.1 \%$ to $27 \%$ mutant allele frequency (CCF $4 \%$, coverage 47 and CCF $55 \%$, coverage 37 ) during the treatment-free interval, but dropped below the detection limit after first treatment (coverage 56). In addition, the major clone at the first time point characterized by MARK2 (CCF 37\%, coverage 74) without treatment ("untreated") was slightly less prominent at the second time point without treatment (CCF $34 \%$, coverage 54 ) and undetectable after treatment (coverage 60). These observations indicate a gradual change in clonal composition during an untreated phase of 6 years and a significant clonal replacement after treatment.

\section{Refractory chronic lymphocytic leukemia is associated with a branched evolution of leukemic cells}

In order to group observed clonal changes into different patterns of evolution, we analyzed overall AF changes between all possible pairs of consecutive samples that we grouped into disease phases for different types of evolution. On the basis of time-dependent changes of CCF, when significant AF changes were unidirectional, these evolution patterns were classified as co-evolution (also termed "linear evolution"). ${ }^{22}$ In contrast, evolution was classified as "branched" when different significant changes concomitantly increased and

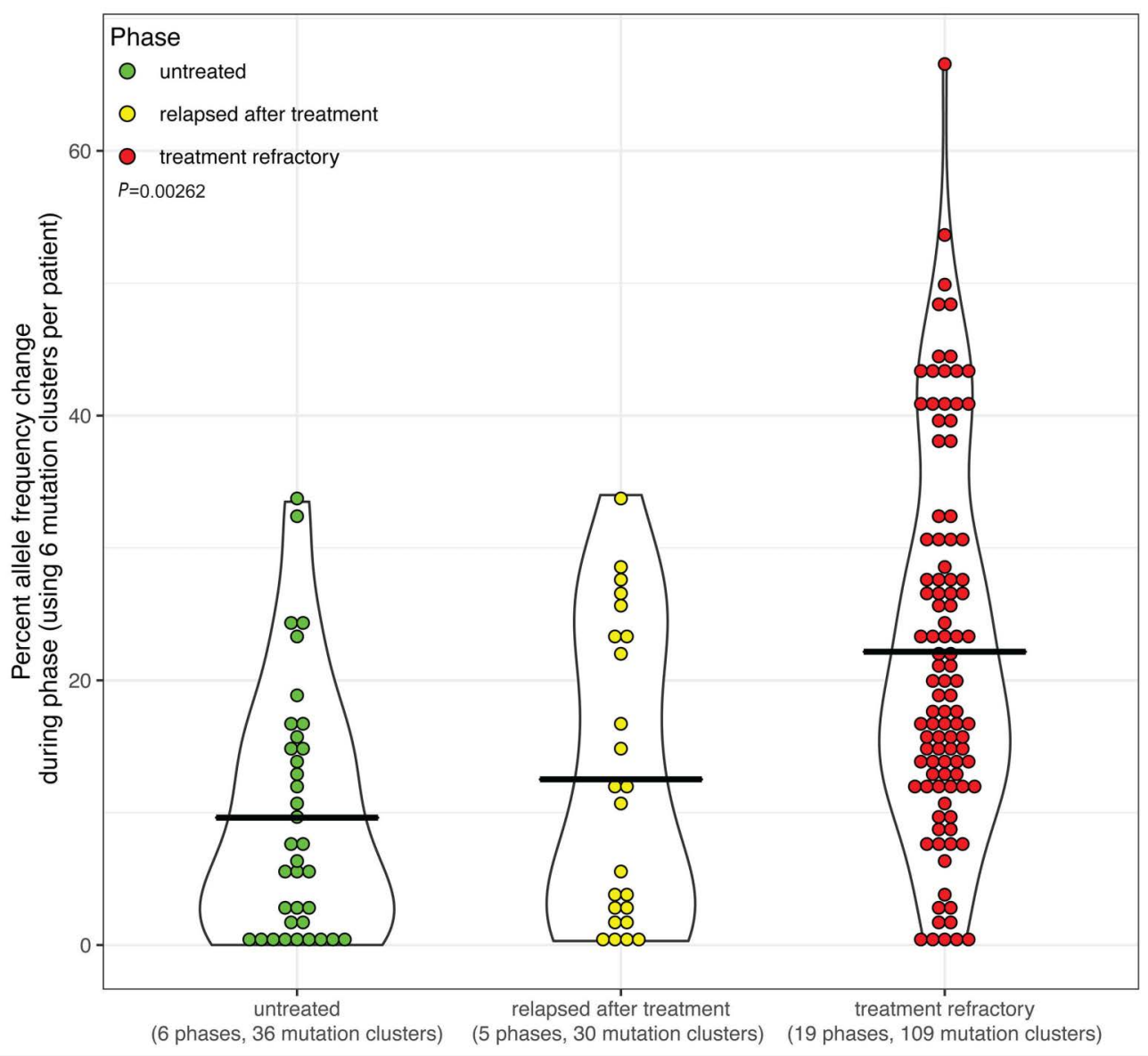

Figure 3. Allele frequency changes across phases. Between two consecutive samplings in a patient, changes in allele frequency of clustered somatic single nucleotide polymorphism (SNP) shown as circles. Circles are grouped according to the clinical phase at the second time point. In order to weigh each patient identically, regardless of the number of mutations, the changes in allele frequency of all single nucleotide variants (SNV) were clustered into six groups per patient and the average of these groups is depicted resulting in six circles per patient phase. Black line represents phase mean. Statistical significance of allele frequency change differences between clinical phases was tested using Kruskal-Wallis test and $P$-value corrected using 100,000 permutations of the phase labels for the six mutation clusters representing each patient $(P=0.00262)$. 
A
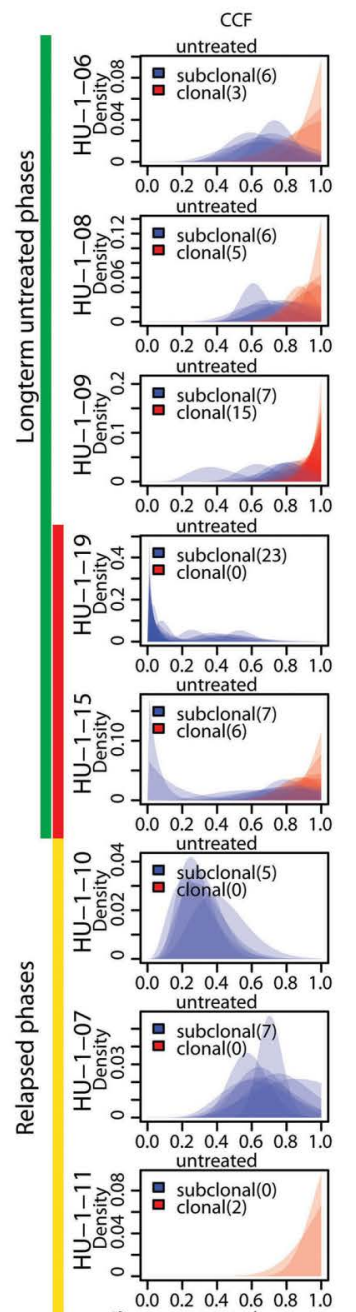

$\begin{array}{lllllllll}0.0 & 0.2 & 0.4 & 0.6 & 0.8 & 1 & 1\end{array}$

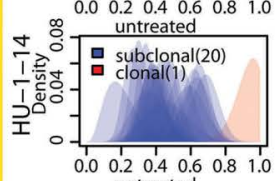

$\begin{array}{llllllll}0.0 & 0.2 & 0.4 & 0.6 & 1 & 0.8 & 1.0\end{array}$

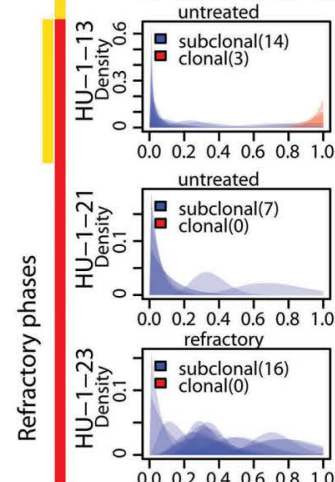

$0.00 .20 .40 .6 \quad 0.81 .0$
CCF

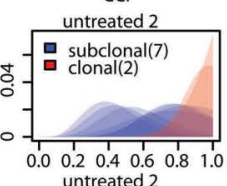

untreated 2

$-$

$\begin{array}{llllllll}0.0 & 0.2 & 0.4 & 0.6 & 0.8 & 1.0\end{array}$

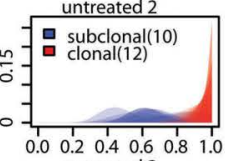

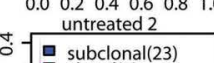

a clonal(0)

$-$

$\begin{array}{llllllll}0.0 & 0.2 & 0.4 & 0.6 & 0.8 & 1.0\end{array}$

$0.0 \quad 0.4 \quad 0.60 .8$
untreated 2

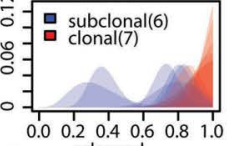

$\begin{array}{lllllll}0.0 & 0.2 & 0.4 & 0.6 & 0.8 & 1.0\end{array}$

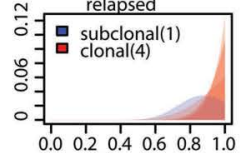

$\begin{array}{cccc}0.0 & 0.2 & 0.4 & 1 \\ \text { relapsed } & 0.6\end{array}$

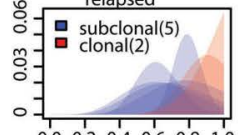

$\begin{array}{llllllllllllll}0.0 & 0.2 & 0.4 & 0.6 & 0.8 & 1.0\end{array}$

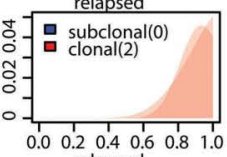

$\begin{array}{lllllll}0.0 & 0.2 & 0.4 & 0.6 & 0.8 & 1.0\end{array}$
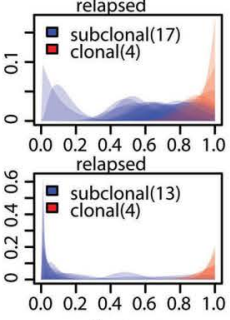

$\begin{array}{lllllll}0.0 & 0.2 & 0.4 & 0.6 & 0.8 & 1.0\end{array}$

$\mathrm{m}-1$ refractory

ำ-

○-

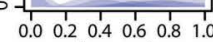$$
\begin{gathered}
0.00 .20 .4 \quad 0.6 \quad 0.81 \\
\text { refractory } 2
\end{gathered}
$$

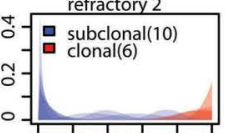

\begin{tabular}{llllllll}
\hline & 1 & 1 & 1 & 1 \\
0.0 & 0.2 & 0.4 & 0.6 & 0.8 & 1.0
\end{tabular}
CCF

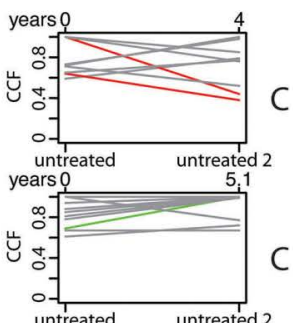

untreated untreate

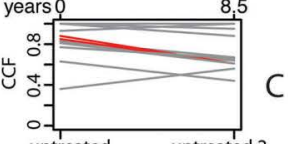

refractory

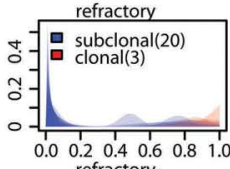

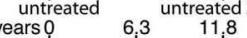

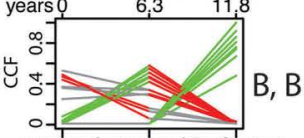

refractory

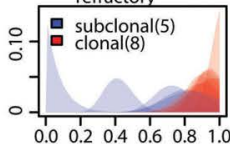

untreated untreated 2 refractory

$\begin{array}{lll}\text { years } 0 & 5,2 & 5\end{array}$

㟧

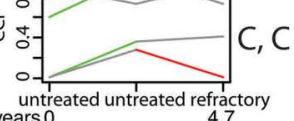

untreated untreated refracto

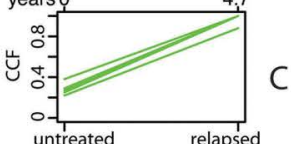

untreated relapsed

years 0

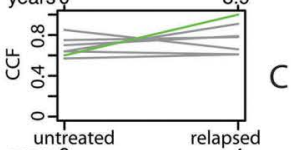

untreated relapsed
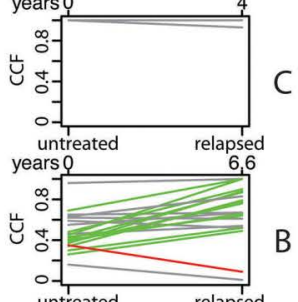

refractory

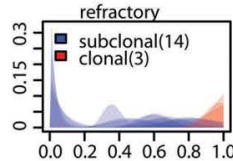

$\begin{array}{lll}\text { untreated } & & \text { relapsed } \\ \text { years } 0 & 8.8 & 11.1\end{array}$

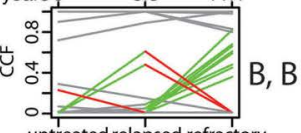

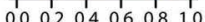

untreated relapsed refractory
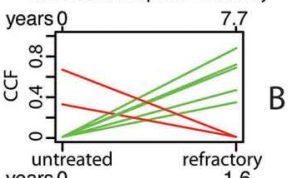

years 0

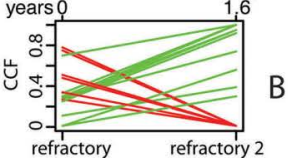

Figure 4. Changes in cancer cell fractions and evolution types. (A) Evolution patterns in 12 patients. Probability distribution of the cancer cell fraction (CCF) for each somatic single nucleotide variant (SNV) revealed clonal (red) or subclonal (blue) SNV (left side). The changes in CCF are depicted in the last column. Changes (a mutation with a change in CCF of greater than $0.2(\Delta \mathrm{CCF}>0.2)$ with probability $>0.5)$ are highlighted in green (increased CCF) or red (reduced CCF). On the basis of time dependent changes of CCF (right side), evolution patterns were considered as (unbranched) co-evolution (C) when significant changes were unidirectional (up or down), or branched (B) when significant changes were in both directions (up and down) indicating that a dominant clone is replaced by its siblings. Time between samplings is indicated in years at the top. (B) Difference in occurrence of evolution types across clinical phases (coevolution = blue, branched evolution $=$ brown)

Mutation type $\square$ clonal $\square$ subclonal CCF change $\square$ increased $\square$ decreased $\square$ not significant

B

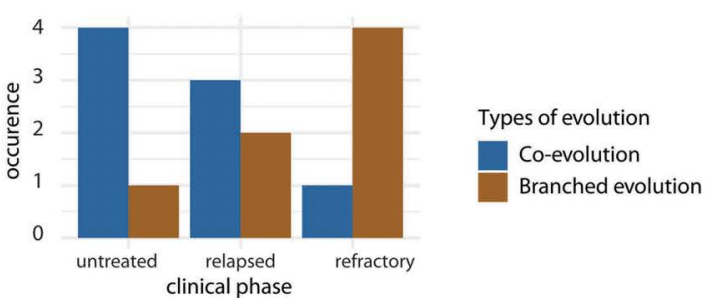


decreased in CCF between consecutive samplings and were thus "bidirectional".

As depicted in Figure 4A, we observed in the group of long-term untreated patients phases in the clinical course where substantial shifts in the clonal composition occurred only after treatment. These shifts resulted either in co-evolution of few sub-clones while other sub-clones were lost (e.g., HU-1-15), or a simultaneous decrease and increase of different subclones indicative of branched evolution. Longterm untreated phases display mainly co-evolution patterns (four of five), while only one patient (HU-1-19), who required treatment after 6 years, followed a branched evolution pattern and became refractory to a subsequent treatment. In phases preceding relapse, co-evolution appeared less frequent (three of five). Instead, relapsed and refractory CLL showed a tendency towards more frequent branched evolution than untreated CLL (six of ten vs. one of five, Fisher exact test $P=0.28$ ), a pattern that was e.g., observed in the relapsed and treatment refractory phases of a single patient (HU-1-13). Interestingly, this patient was treated with FCR (fludarabine, cyclophosphamide, rituximab) in 2005 and in 2009 again achieving a complete response (CR) each time, but at the second time with shorter duration.
The differences in evolution types shows a trend towards more branched evolution in relapsed and even more in refractory phases (Figure 4B). In order to assess the dynamics on a cellular level we inferred the clonal composition based on the cancer cell fractions using TrAP (Figure 5). Interestingly the major subclone at refractory time point in HU-1-19 (clone 3, 41.5\% clone fraction) is already present to a minor extend at the second untreated time point (clone fraction $2.0 \%$ ) whereas the major clone at the first time point further evolved gaining an additional set of mutations subdividing into clones 4,5 and 6 (clone fraction of $13.7 \%, 21.3 \%$ and $10.5 \%)$. In contrast a clone $(19.0 \%$ clone fraction) present at the second untreated time point defined by an EGR2 mutation was undetected at the other last time point. In order to confirm the evolutionary changes with an additional method, we performed epigenetic analysis of 20 samples corresponding to ten phases. In line with the genetic data, large-scale evolution of methylation patterns was not present in any of the evaluated long-term untreated $(n=3)$ and relapsed $(n=2)$ phases displaying clonal changes of linear type while three of the five examined refractory phases featured profound changes in DNA methylation (Online Supplementary Figure
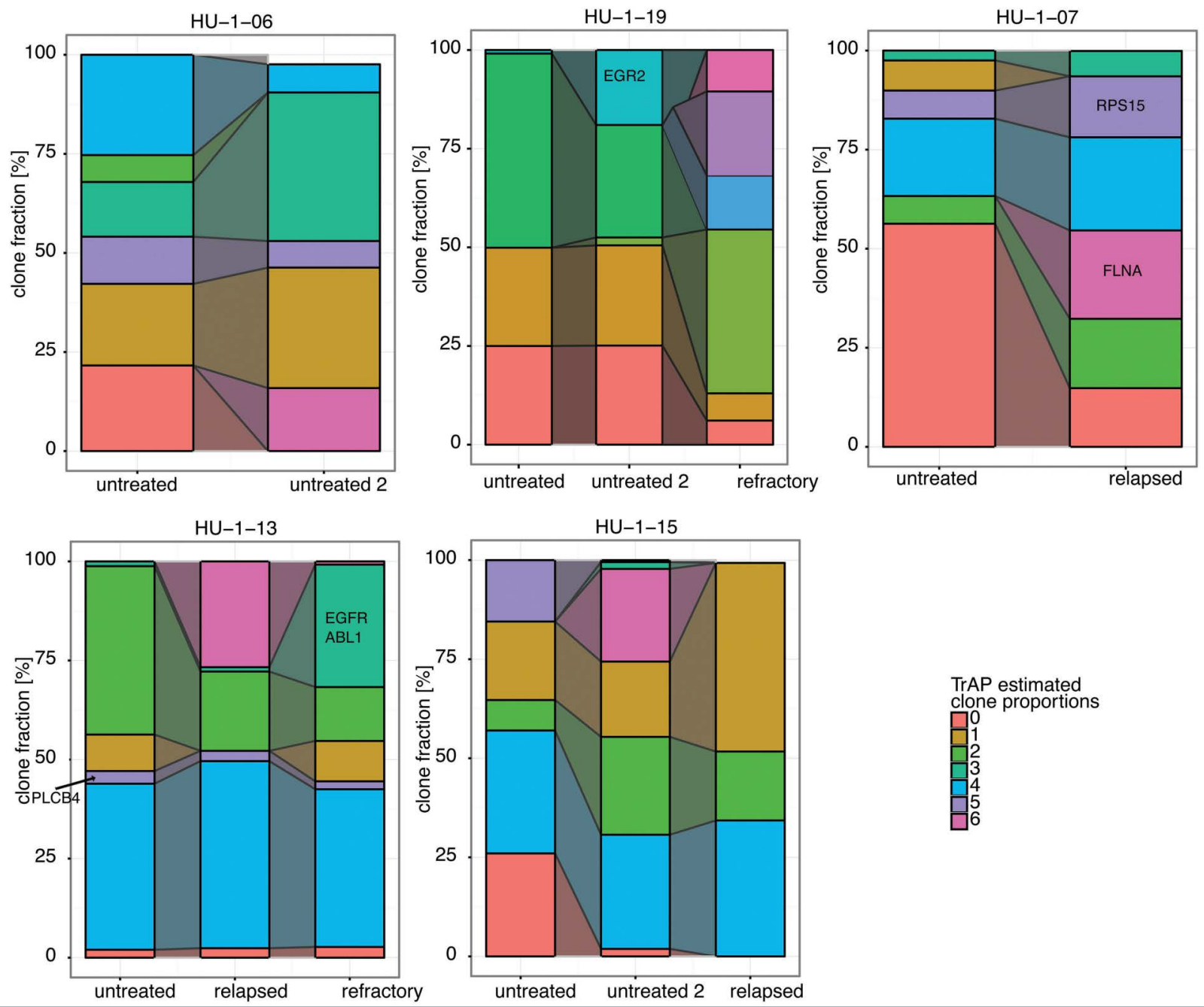

Figure 5. Clonal composition as inferred by TrAP. Exemplary changes in clone fraction for individual patients across samplings. Clonal composition was estimated by applying TrAP on the cancer cell fraction calculated for individual single nucleotide variants (SNV). Different clones are represented by the respective colors and individual time points are indicated on the x-axis. Highlighted with the respective gene symbols are inferred clones linked to a known cancer driver gene. $y$-axis represents clonal fraction of the individual clones identified for the best fit TrAP solution. 
S5). In all patients, even in the ones with few methylation changes, hypermethylation was concentrated in poised promoters and polycomb-repressed regions, whereas hypomethylation occurred mostly in heterochromatin (data not shown; assignment of chromatin states was according to the published reference epigenome of $\mathrm{CLL}^{35}$ ). In spite of this common pattern, we could not identify any specific CpGs that consistently changed the methylation status throughout different patients or phases.

In summary, based on the patients analyzed here, the clonal evolution pattern seems to be linked to the disease phases, and increased changes in AF and a branched evolution are significantly more frequent after treatment compared to untreated patient phases (Figure 6).

\section{Discussion}

Medical history and disease course of patients with CLL is very individual. In this study, we examined WES data of CLL patients acquired at several time points during their disease and treatment course. Comparing consecutive samples from individual patients, we identified somatic mutations that were present in the leukemia cells and tracked over time the changes in AF of these mutations and the underlying fraction of cancer cells that carried the respective mutations. By modeling the clonal composition using the software $\operatorname{TrAP},{ }^{32}$ we discovered different clonal evolution patterns and disease progression courses that were linked to the treatment and response history of the patients (Figure 5). From the mutations and clonal changes that occur during CLL disease progression, we draw the following conclusions with respect to groups of genes, but also more conceptually with respect to clonal composition and evolution over time.

Recurrent mutations in genes were linked to CLL relapse in three different time- and treatment-dependent patterns. ${ }^{36}$ First, one subset of genes initially displays subclonal mutations that are enriched after therapy. In contrast, mutations in a second set of genes remained clonally stable upon relapse. Finally, mutations in a third set of genes that are stable in most patients show clonal enrichment only in rare cases. However, these groups of mutations were not linked to a clinical phenotype. Furthermore, exponential-like growth patterns were recently associated with a larger number of CLL drivers and short time to first treatment..$^{37}$ Of note, these data were derived from untreated CLL patients followed over time. In our patient cohort under the selective pressure of treatment, neither common genetic risk factors like IGHV or recurrent aberrations, nor variants or typically affected pathways are characteristic for a specific clinical course. And although the number of mutations increased slightly after treatment, this did not reflect or even predict outcome after therapy, nor did the number of (sub-)clones. Furthermore, clonal evolution was associated with treatment and indeed branched evolution was found more often in refractory cases, but not exclusively. These results reflect published data for relapsed cases after FCR therapy, which could also not link progression-free survival to an evolution pattern after FC(R) therapy., ${ }^{4,2}$ Dividing our patient groups in long term responder and refractory cases allowed us in contrast to prior attempts to match the duration of response to the extent of the clonal shift. Counterintuitively, clonal evolution that was mostly dynamic and occurred primarily in patients who displayed refractory disease, i.e., where major changes in clonal evolution happened under the guise of a clinically stable or progressing disease. Therefore, what correlated most with the duration of response to treatment was a highly dynamic evolutionary change among sub-clones, and this change was directly associated with refractory disease. In contrast and unexpectedly, relapse after initially durable response occurred mostly with the same sub-clones. We identified three distinctly different courses of clonal evolution that occurred under distinctly different treatment and response patterns. In refractory cases, clonal composition changed dramatically upon treatment failure and in patients 2,4 , and 18 this happened within only 3 months of therapy. Furthermore, in refractory phases, change in clonal composition was often accompanied by a profound shift in the bulk DNA methylation profile of the tumor, most probably reflecting different methylation profiles of the competing clones rather than de novo methylation changes, as it was previously shown that established CLL clones are epigenetically stable and changes in DNA methylation are unlikely to occur without genetic evolution. ${ }^{24}$ As an example, patient HU-1-23 did not gain any new mutations between the two time points of his refractory phase but underwent selection of particular pre-existent CLL clones according to the branched genetic evolution model and this was also manifested by a shift in DNA methylation of the bulk tumor. For the clinician managing the patient, "hidden" selection of a resistant clone is masked by a tumor with a seemingly stable clinical phenotype, i.e., with a persistent lymphadenopathy and leukocytosis. This dynamic clonal change suggests either

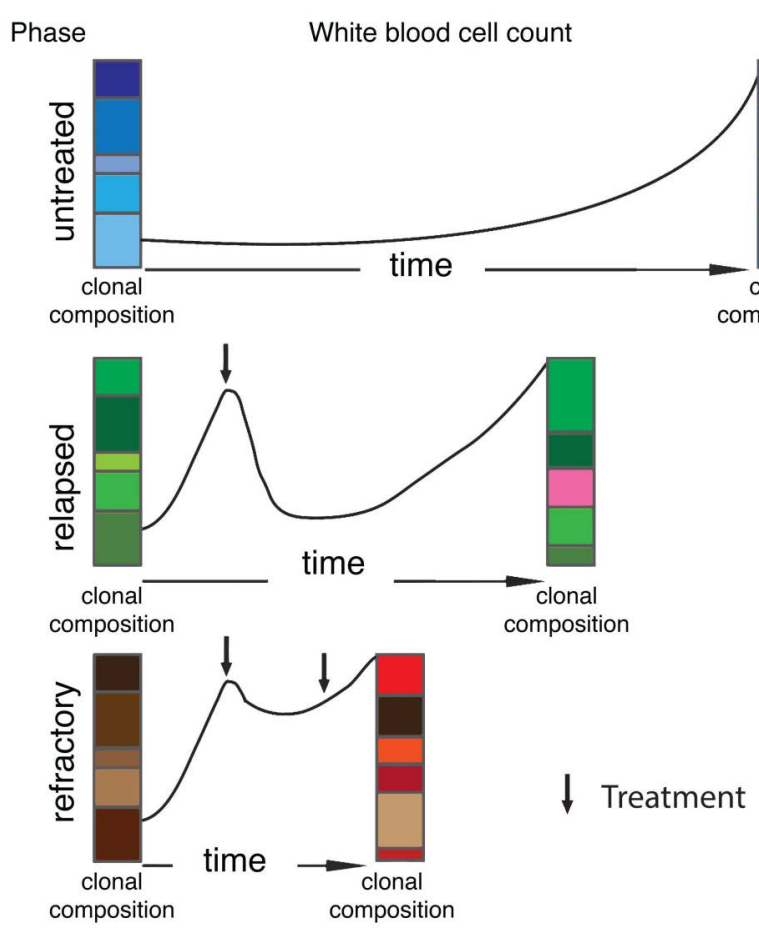

Figure 6. Model of the clonal composition changes. Model of the clonal composition changes in the three different treatment phases (long-term untreated, relapsed and treatment refractory). Black lines indicate lymphocyte counts as surrogate marker for tumor load. Arrows indicate times of treatment. The stacked bar plot indicates clonal tumor composition where different colors indicate a different clone defined by a set of mutations. 
an increased evolutionary capacity in these patients or simply the presence of at least one resilient clone. Importantly a selective pressure of therapy is necessary to induce or catalyze this clonal change as provided by the clinical course of patients 15 and 19. Both have a long term untreated phase without marked evolution but a strong shift after becoming refractory. Therefore, neither the underlying risk factors, the natural disease biology nor the type of evolution, which is branched in untreated and refractory phase in both patients, reflects the clinical course while the extent of evolution does. Importantly, we could show in addition that this process occurs also independently of TP53 mutations, i.e., in a cohort of CLL patients without TP53 aberrations before treatment. Thus, in these patients the clonal evolution is driven mostly by treatment that seems to select for resistant CLL clones.

Thus, our key finding is the striking observation of a clonal turnover during therapy in those patients, who were considered treatment refractory and therefore are assumed to have a stable tumor load.

In contrast, long term untreated cases and late relapses are genomically stable, although they are observed over a much longer period of time. In the latter we found a remarkably stable genomic landscape considering that these patients received a therapy with a subsequent regrowing after a prolonged treatment-free interval. This stability is completely different to a tumor that is refractory and apparently unaffected by therapy, but in contrast displays a dramatic change in clonal composition. This opposing clinical and genomic phenotype at first appears counterintuitive. However, these different courses of clonal dynamics in relapsing and refractory patient phases could be explained by the preexistence of a resistant clone that after removal of the bulk tumor by a treatment intervention will quickly grow out and fill the empty niche. If such resistant clones are absent, competition and outgrowth over time is still possible, so that the tumor regrows with an almost identical clonal composition. This finding mechanistically explains and underlines the relevance of the widely used clinical paradigm of repeating the previous treatment regimen when a good and long-lasting response is achieved: based on the same clonal composition at relapse, the clinician can expect another good response of the tumor to the treatment as the tumor has the same clonal composition as before the treatment. Interestingly, new treatment modalities like venetoclax may behave similarly due to the strong reduction of the tumor load, comparable to chemotherapy: while patients treated with short and effective venetoclax containing combination therapies lack BCL2 mutations at relapse, refractoriness to a long lasting venetoclax treatment associates with the outgrowth of a BCL2 mutated clone displaying the same clonal shift towards drug resistance, that we observe here in chemotherapy refractory cases. ${ }^{38,39}$ On the other hand, ibrutinib may cause a more decelerated clonal shift due to its slow debulking treatment effect and also only slowly emerging resistant clones (i.e., point mutations in BTK/PLCG $2^{40}$ ).
In summary despite the small patient cohort ( $\mathrm{n}=25$ with 54 time points), we identified a link between changes in the variant AF and changes in clonal architecture, both of which are linked with shortened time to further treatment, i.e., treatment resistance. We found clonal evolution to occur without strong contribution of known CLL driver genes. However, there is a dramatic difference in clonal evolution patterns between relapsed and refractory samples, which highlights the importance of the treatment-induced clonal changes in relation to treatment response. This intrinsic characteristic of CLL evolution underlines the relevance of comparing the benefits of treatment compared to the watch-and-wait strategy that has a very low clonal evolution rate. Furthermore, the substantial clonal evolution in refractory disease highlights the need for novel, non-genotoxic treatment regimens with targeted therapy that are less likely to induce clinical disease resistance by selecting out preexistent refractory sub-clones.

\section{Disclosures and Funding}

DM was supported by DFG (SFB1074 subproject B1 and B2) and ERA-NET "FIRE-CLL"; MZ and MS were supported by ERA-NET "FIRE-CLL" and BMBF "PRECISE"; SS received support from DFG (SFB1074 subproject B1 and B2, BMBF "PRECISE" and ERA-NET "FIRE CLL", he also received honoraria and research support from AbbVie, AstraZeneca, Celgene, Gilead, GSK, Hoffmann La-Roche, Janssen, Novartis; ET received honoraria from the Speakers Bureau, Advisory board and travel support of Hoffmann LaRoche and AbbVie.

\section{Contributions}

$P L$ and SS developed concepts and ideas; $M Z$ developed software; ET applied methodology; ET validated data; MZ, ET and $D Y Y$ performed formal analysis; $M Z, E T$ and $S O ̈$ performed investigations; $T Z, C S, J B, H D, E T, P L$ and $S S$ provided resources; $M Z, E T$, and $S S$ cured data; $M Z, E T, S O ̈, M S, D M$, $P L$ and $S S$ wrote the original draft; $M Z, E T, S O ̈, D Y Y, M S$, $T Z, C S, J B, D M, P L$ and $S S$ wrote, reviewed and edited the manuscript; $M Z, E T, S O ̈, D Y Y$ and DM visualized concepts and data; PL and SS supervised the project; PL and SS were in charge of project administration; $M Z, E T, T Z, P L$ and $S S$ acquired funding.

\section{Acknowledgements}

We thank all patients and physicians, especially Andrea Schnaiter, for donating samples and participating in this study. We thank Michael Hain and Rolf Kabbe for computational support. We thank Stephan Wolf and the High Throughput Sequencing unit of the Genomics \& Proteomics Core Facility, German Cancer Research Center (DKFZ), for providing excellent sequencing services.

\section{Data availability}

Sequencing data have been deposited at the European Genome-Phenome Archive hosted at the EBI under accession EGAS00001003652. Data for the methylation arrays are accessible at GSE143411. 


\section{References}

1. McGranahan N, Rosenthal R, Hiley CT, et al. Allele-specific HLA loss and immune escape in lung cancer evolution. Cell. 2017:171(6):1259-1271

2. Nowell PC. The clonal evolution of tumor cell populations. Science. 1976;194(4260): 23-28.

3. Landau DA, Sun C, Rosebrock D, et al. The evolutionary landscape of chronic lymphocytic leukemia treated with ibrutinib targeted therapy. Nat Commun. 2017;8(1):2185.

4. Landau DA, Tausch E, Taylor-Weiner AN, et al. Mutations driving CLL and their evolution in progression and relapse. Nature. 2015;526(7574):525-530.

5. Hallek M, Shanafelt TD, Eichhorst B. Chronic lymphocytic leukaemia. Lancet. 2018;391(10129):1524-1537.

6. Rossi D, Rasi S, Fabbri G, et al. Mutations of NOTCH1 are an independent predictor of survival in chronic lymphocytic leukemia. Blood. 2012;119(2):521-29.

7. Rose-Zerilli MJJ, Forster J, Parker $\mathrm{H}$, et al. The correlation between deletion architecture, ATM mutational status and BIRC3 disruption in 11q-deleted CLL. Blood. 2012;120(21):658-658.

8. Stilgenbauer S, Schnaiter A, Paschka P, et al. Gene mutations and treatment outcome in chronic lymphocytic leukemia: results from the CLL8 trial. Blood. 2014;123(21):32473254.

9. Puente XS, Bea S, Valdes-Mas R, et al. Noncoding recurrent mutations in chronic lymphocytic leukaemia. Nature. 2015;526 (7574):519-524.

10. Dohner H, Stilgenbauer S, Benner A, et al. Genomic aberrations and survival in chronic lymphocytic leukemia. N Engl J Med. 2000;343(26):1910-1916

11. Zenz T, Eichhorst B, Busch R, et al. TP53 mutation and survival in chronic lymphocytic leukemia. J Clin Oncol. 2010;28(29): 4473-4479.

12. Schnaiter A, Paschka P, Rossi M, et al. NOTCH1, SF3B1, and TP53 mutations in fludarabine-refractory CLL patients treated with alemtuzumab: results from the CLL2H trial of the GCLLSG. Blood. 2013;122(7): 1266-1270

13. Winkelmann N, Rose-Zerilli M, Forster J, et al. Low frequency mutations independently predict poor treatment-free survival in early stage chronic lymphocytic leukemia and monoclonal B-cell lymphocytosis. Haematologica. 2015;100(6):e237-239.

14. Han T, Ohtaki K, Sadamori N, et al. Cytogenetic evidence for clonal evolution in
B-cell chronic lymphocytic leukemia. Cancer Genet Cytogenet. 1986;23(4):321-328.

15. Oscier D, Fitchett M, Herbert T, Lambert R. Karyotypic evolution in B-cell chronic lymphocytic leukaemia. Genes Chromosomes Cancer. 1991;3(1):16-20.

16. Shanafelt TD, Witzig TE, Fink SR, et al. Prospective evaluation of clonal evolution during long-term follow-up of patients with untreated early-stage chronic lymphocytic leukemia. J Clin Oncol. 2006;24(28):46344641.

17. Stilgenbauer S, Sander S, Bullinger L, et al. Clonal evolution in chronic lymphocytic leukemia: acquisition of high-risk genomic aberrations associated with unmutated $\mathrm{VH}$, resistance to therapy, and short survival. Haematologica. 2007;92(9):1242-1245.

18. Knight SJ, Yau C, Clifford R, et al. Quantification of subclonal distributions of recurrent genomic aberrations in paired pretreatment and relapse samples from patients with B-cell chronic lymphocytic leukemia. Leukemia. 2012;26(7):1564-1575

19. Puente XS, Pinyol M, Quesada V, et al. Whole-genome sequencing identifies recurrent mutations in chronic lymphocytic leukaemia. Nature. 2011:475(7354):101-105.

20. Landau DA, Wu CJ. Chronic lymphocytic leukemia: molecular heterogeneity revealed by high-throughput genomics. Genome Med. 2013;5(5):47.

21. Schuh A, Becq J, Humphray S, et al. Monitoring chronic lymphocytic leukemia progression by whole genome sequencing reveals heterogeneous clonal evolution patterns. Blood. 2012;120(20):4191-4196.

22. Landau DA, Carter SL, Stojanov P, et al. Evolution and impact of subclonal mutations in chronic lymphocytic leukemia. Cell. 2013;152(4):714-726.

23. Ojha J, Ayres J, Secreto C, et al. Deep sequencing identifies genetic heterogeneity and recurrent convergent evolution in chronic lymphocytic leukemia. Blood. 2015;125(3):492-498.

24. Oakes CC, Claus R, Gu L, et al. Evolution of DNA methylation is linked to genetic aberrations in chronic lymphocytic leukemia. Cancer Discov. 2014;4(3):348-361.

25. Rasi S, Khiabanian H, Ciardullo C, et al. Clinical impact of small subclones harboring NOTCH1, SF3B1 or BIRC3 mutations in chronic lymphocytic leukemia. Haematologica, 2016;101(4):135-138.

26. Nadeu F, Delgado J, Royo C, et al. Clinical impact of clonal and subclonal TP53, SF3B1, BIRC3, NOTCH1, and ATM mutations in chronic lymphocytic leukemia. Blood. 2016;127(17):2122-2130.

27. Nadeu F, Clot G, Delgado J, et al. Clinical impact of the subclonal architecture and mutational complexity in chronic lymphocytic leukemia. Leukemia. 2018;32(3):645653.

28. Wang J, Khiabanian H, Rossi D, et al. Tumor evolutionary directed graphs and the history of chronic lymphocytic leukemia. Elife. 2014;3:e02869.

29. Jones DT, Hutter B, Jager N, et al. Recurrent somatic alterations of FGFR1 and NTRK2 in pilocytic astrocytoma. Nat Genet. 2013;45 (8):927-932.

30. Koboldt DC, Zhang Q, Larson DE, et al. VarScan 2: somatic mutation and copy number alteration discovery in cancer by exome sequencing. Genome Res. 2012;22(3):568576

31. Carter SL, Cibulskis K, Helman E, et al. Absolute quantification of somatic DNA alterations in human cancer. Nat Biotechnol. 2012;30(5):413-421.

32. Strino F, Parisi F, Micsinai M, Kluger Y. TrAp a tree approach for fingerprinting subclonal tumor composition. Nucleic Acids Res. 2013;41(17):e165.

33. Jones DT, Jager N, Kool M, et al. Dissecting the genomic complexity underlying medulloblastoma. Nature. 2012;488(7409):100105

34. Bailey MH, Tokheim C, Porta-Pardo E, et al. Comprehensive characterization of cancer driver genes and mutations. Cell. 2018;173 (2):371-385

35. Beekman R, Chapaprieta V, Russinol N, et al. The reference epigenome and regulatory chromatin landscape of chronic lymphocytic leukemia. Nat Med. 2018;24(6):868-880.

36. Amin NA, Seymour E, Saiya-Cork K, Parkin B, Shedden K, Malek SN. A Quantitative analysis of subclonal and clonal gene mutations before and after therapy in chronic lymphocytic leukemia. Clin Cancer Res. 2016;22(17):4525-4535.

37. Gruber M, Bozic I, Leshchiner I, et al Growth dynamics in naturally progressing chronic lymphocytic leukaemia. Nature. 2019;570(7762):474-479.

38. Tausch E, Close W, Dolnik A, et al Venetoclax resistance and acquired BCL2 mutations in chronic lymphocytic leukemia. Haematologica. 2019;104(9):e434-e437.

39. Blombery P, Anderson MA, Gong JN, et al Acquisition of the recurrent Gly101Val mutation in BCL2 confers resistance to venetoclax in patients with progressive chronic lymphocytic leukemia. Cancer Discov. 2019;9(3):342-353

40. Woyach JA, Furman RR, Liu TM, et al. Resistance mechanisms for the Bruton's tyrosine kinase inhibitor ibrutinib. N Engl J Med. 2014;370(24):2286-2294. 